\section{High Precise Fuzzy Control for Piezoelectric Direct Drive Electro-Hydraulic Servo Valve*}

\author{
Miaolei ZHOU**, Wei GAO**, Zhigang YANG*** and Yantao TIAN** \\ ${ }^{* *}$ College of Communication Engineering, Jilin University, \\ Changchun 130022, China \\ E-mail: zml@jlu.edu.cn \\ ${ }^{* *}$ College of mechanical science and Engineering, Jilin University, \\ Changchun 130022, China
}

\begin{abstract}
As the core part of the electro-hydraulic servo system, the electro-hydraulic servo valve plays an important role in the system. Due to the piezoelectric actuator has characteristics of fast response, easy control and high control accuracy, it can be used as the electric-mechanical converter of the electro-hydraulic servo valve. However, this kind of the piezoelectric direct drive electro-hydraulic servo valve has hysteresis and creep nonlinearities. In order to improve the output accuracy of the system, a high-precise fuzzy control method with the dynamic Preisach model in feedforward loop is proposed. The control scheme is separated into two parts: a feedforward loop with the dynamic Preisach model and a feedback loop with high precise fuzzy control. The high precision fuzzy control adopts Lagrange interpolation method. The experimental results show that the proposed method can resolve the hysteresis and creep nonlinearities and reach a higher dynamic performance, the control effect is better than the conventional fuzzy control method.
\end{abstract}

Key words: Piezoelectric, Electro-Hydraulic Servo Valve, Fuzzy Control, Preisach, Hysteresis, Creep

\section{Introduction}

Electro-hydraulic servo system is widely used in machine tools, construction, aerospace and other fields. The electro-hydraulic servo valve is a very important part in the electro-hydraulic servo system. The traditional electro-hydraulic servo valve generally uses torque motor as electric-mechanical converter. Electro-hydraulic servo valve based on this driving mode will lead to low resolution, narrow work bandwidth, and application limits.

Due to the piezoelectric actuator ${ }^{(1)-(3)}$ has the advantages of fast response, easy control and high control accuracy, it can be used as the electric-mechanical converter of electro-hydraulic servo valve ${ }^{(4)-(8)}$. However, the main drawback of the piezoelectric actuator is that there are large hysteresis loops ${ }^{(9)-(11)}$ (up to 15\%) between the input voltage and the output displacement. Therefore, the output of this kind of electro-hydraulic servo valve will produce larger hysteresis nonlinearity when using the piezoelectric actuator to drive electro-hydraulic servo valve. Usually the electro-hydraulic servo valve allows the hysteresis loop not more than $3 \%$. In order to improve the precision of output displacement, the control method that solves this problem needs to be studied.

Ge and Jouaneh ${ }^{(12)}$ presented a computer-based tracking control approach which was based on combining a feedforward loop with a feedback loop in the controller. In the
Received 15 May, 2012 (No. 12-0216) [DOI: 10.1299/jamdsm.6.1154]

Copyright (C) 2012 by JSME 
feedforward loop, Preisach model was adopted to predict the hysteresis nonlinearity. In the feedback loop, the PID controller was adopted to enhance the tracking precision. Zhou et al. (13) proposed a high-precise fuzzy control method with hysteresis nonlinear model in the feedforward loop was proposed. The control scheme was separated into two parts: a feedforward loop with Preisach hysteresis nonlinear model for predicting the hysteresis nonlinearity and a feedback loop with high-precise fuzzy controller for enhance the controlling precision further. Nobuhiko ${ }^{(1)}$ proposed a unique open-loop driving method which was for point-to-point positioning of piezoelectric actuator. In the method, initializing motion was applied in order to make the status of actuator uniform before every stepwise motion. Then the actuator was contracted to a constant position and then elongated to desired position with some overshoot. Micro-meter order positioning accuracy can be obtained by the method. By a modified driving method which is called initializing drive, nano-meter order stepwise positioning for several micro-meter motion range was realized by open-loop drive. Wong et al. ${ }^{(14)}$ proposed two new approaches to model and compensate the rate-dependent hysteresis of a piezostage driven by piezoelectric stack actuators. Online least squares support vector machine (LS-SVM) and online relevance vector machine (RVM) models were used to capture the hysteretic behavior. An inverse model-based feedforward combined with proportional-integral-derivative feedback control was presented. The experiment results showed that the LS-SVM model-based control scheme is much accurate than the RVM model-based one, whereas the latter is much faster than the former in terms of updating time and both of the control schemes could suppress the rate-dependent hysteresis to a negligible level. Song et al. ${ }^{(15)}$ used the inverse Preisach model to eliminate hysteresis nonlinearity and used a cascaded PD/lead-lag feedback controller to improve the control accuracy further. Hwang et al. ${ }^{(16)}$ also proposed a control scheme contained a nonlinear state observer to estimate the unavailable state, a reference model to assign the desired dynamics, and a feedback linearization with variable structure controller.

In this paper, a high-precise fuzzy control method with the dynamic Preisach model in feedforward loop is proposed. The proposed control method has been implemented on a piezoelectric direct drive electro-hydraulic servo valve system. Experimental results have demonstrated high performance of the proposed control method.

\section{The Structure and Characteristics of Piezoelectric Direct Drive Electro-Hydraulic Servo Valve}

Fig. 1 shows the structure of piezoelectric direct drive electro-hydraulic servo valve.

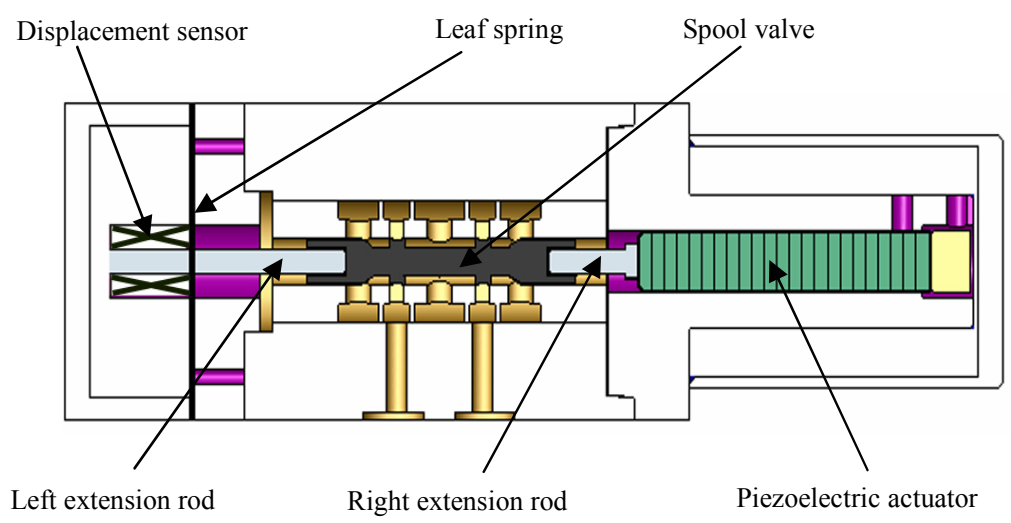

Fig. 1 Structure of piezoelectric direct drive electro-hydraulic servo valve

The displacement of spool valve can be detected by the displacement sensor. When control voltage is applied, the displacement of piezoelectric actuator can make the leaf 
spring deform through the right extension rod, spool valve and left extension rod. Piezoelectric actuator elongates and makes the spool valve move left movement when input voltage increased. The recovery force of leaf spring makes the spool valve move right movement when input voltage decreased. Hence, we can drive the reciprocating motion of spool valve to control the changes of flowrate. The prototype of the piezoelectric direct drive electro-hydraulic servo valve is shown in Fig. 2.

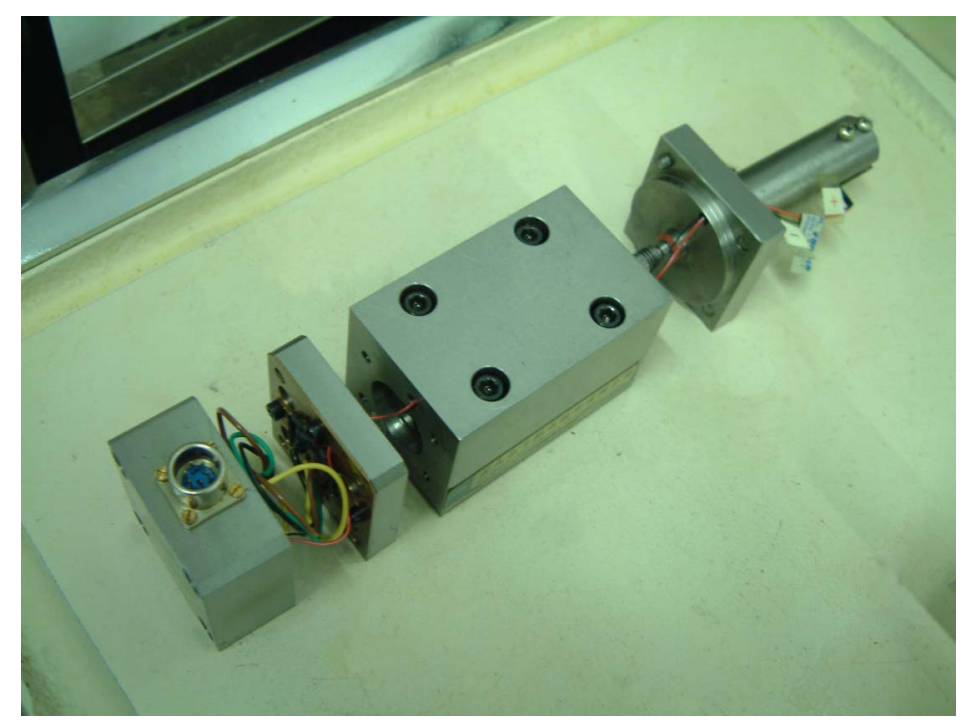

Fig. 2 Piezoelectric direct drive electro-hydraulic servo valve

Fig. 3 shows the input and output characteristic curves of the piezoelectric direct drive electro-hydraulic servo valve. It can be known that the system's output at next moment, not only depends on the current input and output, but also is related to the history of the input value. The output displacement is different under the same input voltage, the increased curve and decreased curve are obviously not coincident with each other. In the stage of voltage decreased, voltage peak is different when returning and the output displacement is also different. This phenomenon presents the hysteresis nonlinearity. This hysteresis characteristic is the main factor that influences the output accuracy of the piezoelectric direct drive electro-hydraulic servo valve.

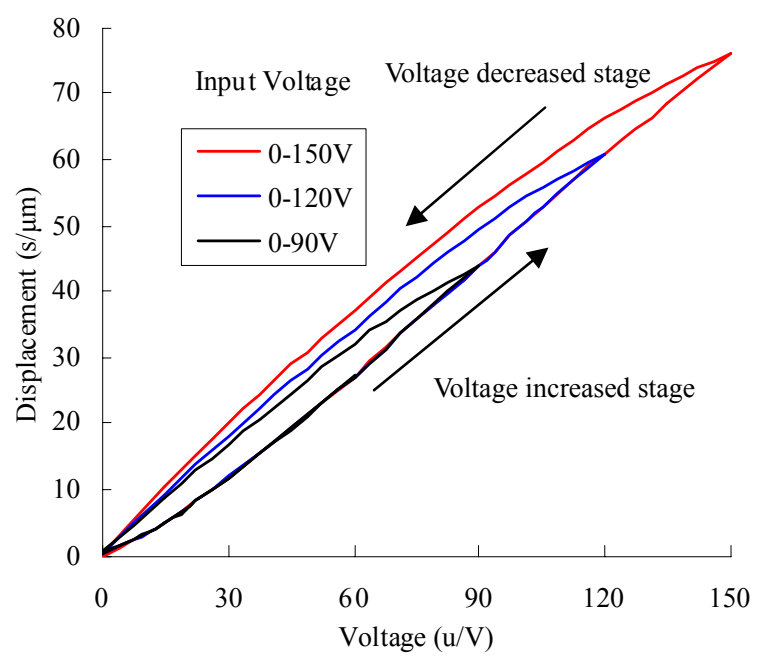

Fig. 3 Hysteresis nonlinearity characteristic curve 
Fig. 4 shows the output displacement of the hysteresis curves of the piezoelectric direct drive electro-hydraulic servo valve with $0.1 \mathrm{~Hz}, 1 \mathrm{~Hz}$ and $10 \mathrm{~Hz}$ voltage exciting signals.

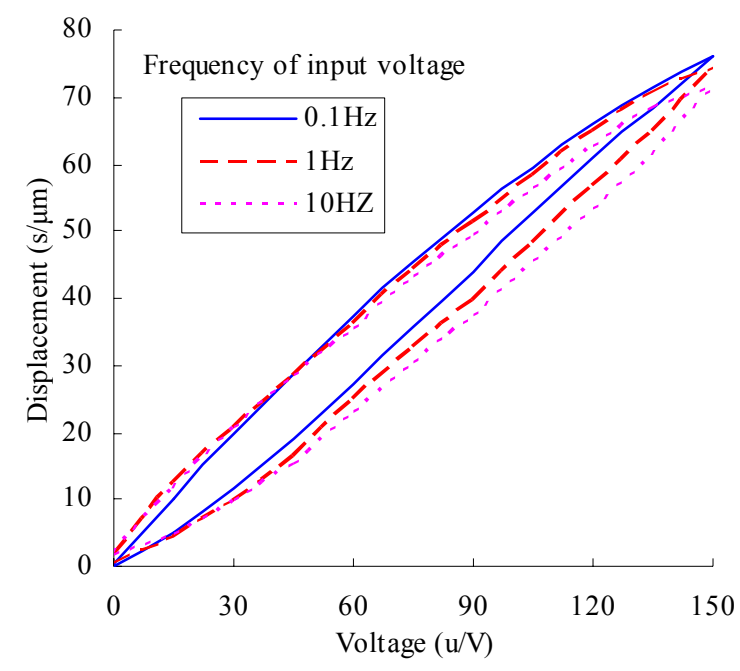

Fig. 4 Hysteresis curves under different frequency exciting signals

It can be seen from Fig. 4, when the exciting frequency is increasing, the hysteresis curve will be inclining. Namely, output displacement is related to the frequency of input voltage. So the hysteresis nonlinearity of the piezoelectric direct drive electro-hydraulic servo valve is rate-dependent.

Fig. 5 shows the displacement of output curve under $90 \mathrm{~V}$ voltage in long time (100 seconds). It can be seen that the output of the system can quickly complete after the control voltage applied ( $\mathrm{ms}$ time level), and for a long time it is a process that output value continues to produce small changes, which is called the nonlinear creep characteristic. This characteristic will have also a certain impact on the control accuracy of the piezoelectric direct drive electro-hydraulic servo valve.

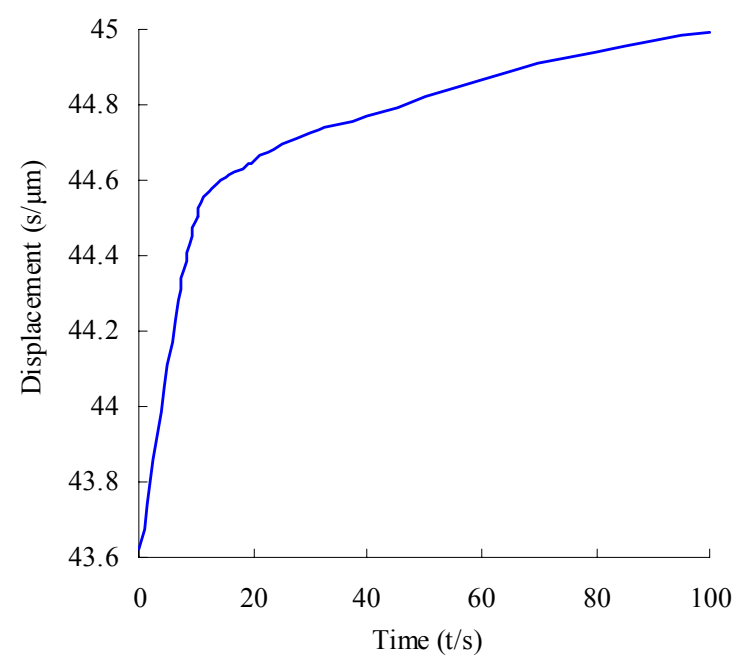

Fig. 5 Creep characteristic curve

From the Figs. 3, 4 and 5, it can be seen that the complex nonlinearities exist in the piezoelectric direct drive electro-hydraulic servo valve system. Therefore we need to 
consider using appropriate control method to improve the accuracy of the system.

\section{Control Scheme}

\subsection{Dynamic Preisach Model for the Piezoelectric Direct Drive Electro-hydraulic Servo Valve}

The conventional Preisach model(CPM) can be represented in mathematical form as follows:

$$
f(t)=\iint_{\alpha \geq \beta} \mu_{0}(\alpha, \beta) \gamma_{\alpha \beta} u(t) d \alpha d \beta=\iint_{S^{+}} \mu_{0}(\alpha, \beta) d \alpha d \beta
$$

where, $\alpha$ and $\beta$ are the switching values of input: $\alpha$ corresponds to the maximum value of input extreme, and $\beta$ corresponds to the minimum value of input extreme; $\mu_{0}(\alpha, \beta)$ is a weight function of the Preisach model; $\gamma_{\alpha \beta} u(t)$ is the hysteresis operator, which has two output values, 0 or 1 .

Function $F$ is defined as follows:

$$
F(\alpha, \beta)=f_{\alpha}-f_{\alpha \beta}
$$

where $f_{\alpha}$ is output value when $u(t)$ rise from 0 to $\alpha, f_{\alpha \beta}$ is output value when control voltage $u(t)$ rise from 0 to $\alpha$ then drop to $\beta$.

Assume that the input $u(t)$ is illustrated by fig. 6 :

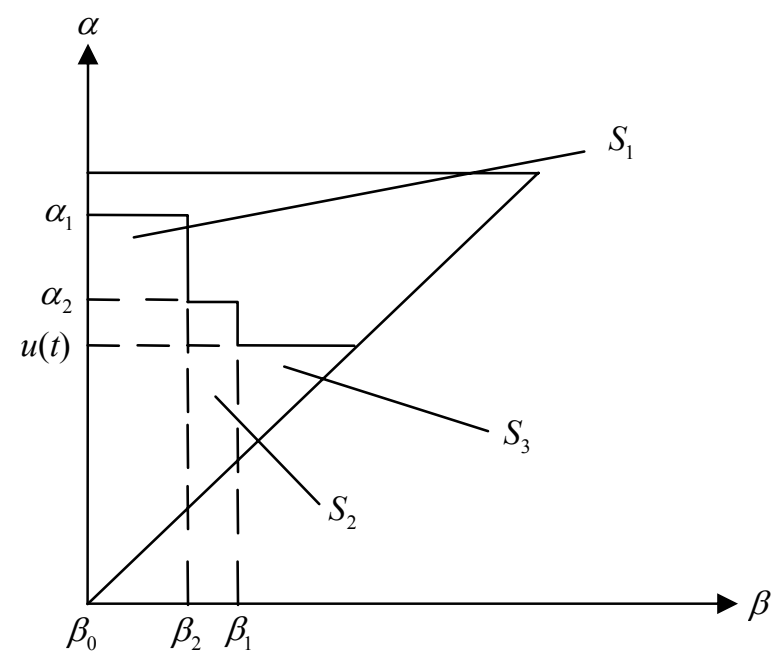

Fig. $6 \quad \alpha-\beta$ plane

$$
\begin{aligned}
\iint_{S^{+}} \mu_{0}(\alpha, \beta) d \alpha d \beta & =\iint_{S_{1}} \mu_{0}(\alpha, \beta) d \alpha d \beta+\iint_{S_{2}} \mu_{0}(\alpha, \beta) d \alpha d \beta+\iint_{S_{3}} \mu_{0}(\alpha, \beta) d \alpha d \beta \\
& =\left[F\left(\alpha_{1}, \beta_{0}\right)-F\left(\alpha_{1}, \beta_{1}\right)\right]+\left[F\left(\alpha_{2}, \beta_{1}\right)-F\left(\alpha_{2}, \beta_{2}\right)\right]+F\left[u(t), \beta_{2}\right]
\end{aligned}
$$

So, numerical implementation of the $\mathrm{CPM}$ is:

$$
f(t)=\sum_{\mathrm{k}=1}^{n}\left[F\left(\alpha_{k}, \beta_{k-1}\right)-F\left(\alpha_{k}, \beta_{k}\right)\right]+F\left(u(t), \beta_{n-1}\right) \quad \dot{u}(t) \geq 0
$$




$$
f(t)=\sum_{\mathrm{k}=1}^{n-1}\left[F\left(\alpha_{k}, \beta_{k-1}\right)-F\left(\alpha_{k}, \beta_{k}\right)\right]+\left[F\left(\alpha_{n}, \beta_{n-1}\right)-F\left(\alpha_{n}, u(t)\right] \quad \dot{u}(t)<0\right.
$$

The traditional Preisach model can express static and rate-independent hysteresis nonlinearity ${ }^{(17)}$, but is difficultly to describe the rate-dependent hysteresis nonlinearity of the piezoelectric direct drive electro-hydraulic servo valve. Therefore, the paper adopted a inverse dynamic Preisach model based on the dynamic Preisach model ${ }^{(18)}$ to solve the control problem of rate-dependent hysteresis nonlinearity of piezoelectric direct drive electro-hydraulic servo valve.

By introducing a function of expressing the rate change in the modified Preisach model, the dynamic Preisach model is described as:

$$
f(t)=\iint_{S^{+}} \mu\left(\alpha, \beta, \chi\left(\frac{d u}{d t}\right)\right) \gamma_{\alpha \beta} u(t) d \alpha d \beta
$$

where, $\chi\left(\frac{d u}{d t}\right)$ is the input change-rate function, it is used to describe the relationship between input change-rate and hysteretic loop.

According to reference(17), Eq. (6) can be written as

$$
f(t)=\iint_{S^{+}} \mu_{0}(\alpha, \beta) d \alpha d \beta+\iint_{S^{+}} \chi\left(\frac{d u}{d t}\right) \mu_{1}(\alpha, \beta) d \alpha d \beta
$$

The positive sets $S^{+}$can be subdivided into n trapezoids $Q_{k}$ with vertices whose $\alpha$ and $\beta$ coordinates are equal to $\left(\alpha_{k}, \beta_{k}\right)$ and $\left(\alpha_{k}, \beta_{k-1}\right)$. Hence, Equation (8) can been obtained

$$
\iint_{S^{+}} \chi\left(\frac{d u}{d t}\right) \mu_{1}(\alpha, \beta) d \alpha d \beta=\sum_{k=1}^{n(t)+1} \iint_{Q_{k}} \chi\left(\frac{d u}{d t}\right) \mu_{1}(\alpha, \beta) d \alpha d \beta
$$

If each trapezoid $Q_{k}$ is divided into $n_{j}^{k}$ sub-trapezoids $q_{j}^{k}$ (see in Fig. 7), then Eq. (8) can be written as

$$
\iint_{S^{+}} \chi\left(\frac{d u}{d t}\right) \mu_{1}(\alpha, \beta) d \alpha d \beta=\sum_{k=1}^{n(t)+1} \sum_{j=1}^{n_{j}^{k}} \iint_{q_{j}^{k}} \chi\left(\frac{d u}{d t}\right) \mu_{1}(\alpha, \beta) d \alpha d \beta
$$

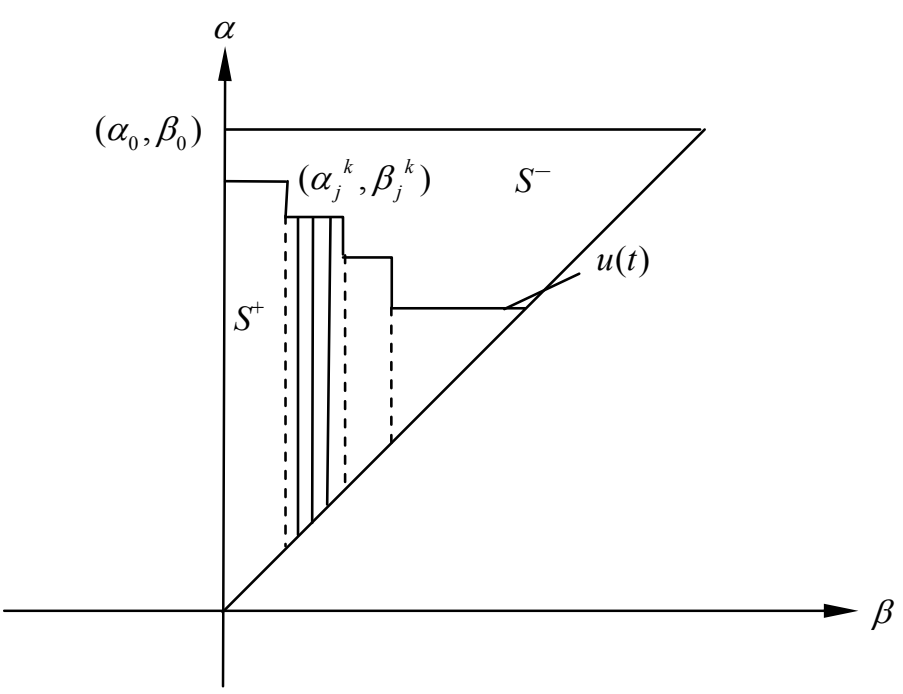

Fig. 7 Each trapezoid $Q_{k}$ divided into $n_{j}^{k}$ sub-trapezoids $q_{j}^{k}$ 
Mean Theorem of Integration is applied on each sub-trapezoid $q_{j}^{k}$ and Eq. (9) can be written as

$$
\iint_{S^{+}} \chi\left(\frac{d u}{d t}\right) \mu_{1}(\alpha, \beta) d \alpha d \beta=\left.\sum_{k=1}^{n(t)+1} \sum_{j=1}^{n_{j}^{k}} \chi\left(\frac{d u}{d t}\right)\right|_{t=\zeta_{j}} \iint_{q_{j}^{k}} \mu_{1}(\alpha, \beta) d \alpha d \beta
$$

where $t=\zeta_{j}$ corresponds to the point of $\alpha, \beta$ in $q_{j}^{k}$, which is determined by the Mean Theorem of Integration.

In the experimental condition, Eq. (9) is also valid for the same sub-trapezoid $q_{j}^{k}$.

Hence

$$
\iint_{q_{j}^{k}} \chi\left(\frac{d u^{e}}{d t}\right) \mu_{1}(\alpha, \beta) d \alpha d \beta=\left.\chi\left(\frac{d u^{e}}{d t}\right)\right|_{t=\zeta_{j}^{e}} \iint_{q_{j}^{k}} \mu_{1}(\alpha, \beta) d \alpha d \beta
$$

According to the definition of $F$ function, Eq. (11) can also be written as

$$
f_{\alpha_{j} \beta_{j-1}}-f_{\alpha_{j} \beta_{j}}=\left.\chi\left(\frac{d u^{e}}{d t}\right)\right|_{t=\zeta_{j}^{e}} \iint_{q_{j}^{k}} \mu_{1}(\alpha, \beta) d \alpha d \beta
$$

Hence

$$
\begin{aligned}
& \iint_{q_{j}^{k}} \mu_{1}(\alpha, \beta) d \alpha d \beta=\frac{1}{\left.\chi\left(\frac{d u^{e}}{d t}\right)\right|_{t=\zeta_{j}^{e}}}\left(f_{\alpha_{j} \beta_{j-1}}-f_{\alpha_{j} \beta j}\right) \\
& \iint_{S_{+}^{+}} \chi\left(\frac{d u}{d t}\right) \mu_{1}(\alpha, \beta) d \alpha d \beta=\sum_{k=1}^{n(t)+1} \sum_{j=1}^{n_{j}^{k}} \frac{\left.\chi\left(\frac{d u}{d t}\right)\right|_{t=\zeta_{j}}}{\left.\chi\left(\frac{d u^{e}}{d t}\right)\right|_{t=\zeta_{j}^{e}}}\left(f_{\alpha_{j} \beta_{j-1}}-f_{\alpha_{j} \beta_{j}}\right)
\end{aligned}
$$

Eq. (14) can be also written as

$$
\begin{gathered}
\iint_{S^{+}} \chi\left(\frac{d u}{d t}\right) \mu_{1}(\alpha, \beta) d \alpha d \beta=\sum_{k=1}^{n(t)} \sum_{j=1}^{n_{j}^{k}} \frac{\left.\chi\left(\frac{d u}{d t}\right)\right|_{t=\zeta_{j}}}{\left.\chi\left(\frac{d u^{e}}{d t}\right)\right|_{t=\zeta_{j}^{e}}}\left(f_{\alpha_{j} \beta_{j-1}}-f_{\alpha_{j} \beta_{j}}\right) \\
+F\left(u(t), \beta_{n-1}\right) \quad \dot{u}(t) \geq 0 \\
\iint_{S_{+}^{+}} \chi\left(\frac{d u}{d t}\right) \mu_{1}(\alpha, \beta) d \alpha d \beta=\sum_{k=1}^{n(t)-1} \sum_{j=1}^{n_{j}^{k}} \frac{\left.\chi\left(\frac{d u}{d t}\right)\right|_{t=\zeta_{j}}}{\left.\chi\left(\frac{d u^{e}}{d t}\right)\right|_{t=\zeta_{j}^{e}}}\left(f_{\alpha_{j} \beta_{j-1}}-f_{\alpha_{j} \beta_{j}}\right) \\
+\left[F\left(\alpha_{n}, \beta_{n-1}\right)-F\left(\alpha_{n}, u(t)\right)\right] \quad \dot{u}(t)<0
\end{gathered}
$$

Hence, numerical implementation of the dynamic Preisach model is

$$
\begin{aligned}
f(t)= & \sum_{\mathrm{k}=1}^{n(t)}\left[F\left(\alpha_{k}, \beta_{k-1}\right)-F\left(\alpha_{k}, \beta_{k}\right)\right]+F\left(u(t), \beta_{n-1}\right) \\
& +\sum_{k=1}^{n(t)} \sum_{j=1}^{n_{j}^{k}} \frac{\left.\chi\left(\frac{d u}{d t}\right)\right|_{t=\zeta_{j}}}{\left.\chi\left(\frac{d u^{e}}{d t}\right)\right|_{t=\zeta_{j}^{e}}}\left(f_{\alpha_{j} \beta_{j-1}}-f_{\alpha_{j} \beta_{j}}\right)+F\left(u(t), \beta_{n-1}\right) \quad \dot{u}(t) \geq 0 \\
f(t)= & \sum_{\mathrm{k}=1}^{n(t)-1}\left[F\left(\alpha_{k}, \beta_{k-1}\right)-F\left(\alpha_{k}, \beta_{k}\right)\right]+\left[F\left(\alpha_{n}, \beta_{n-1}\right)-F\left(\alpha_{n}, u(t)\right)\right]
\end{aligned}
$$




$$
+\sum_{k=1}^{n(t)-1} \sum_{j=1}^{n_{j}^{k}} \frac{\left.\chi\left(\frac{d u}{d t}\right)\right|_{t=\zeta_{j}}}{\left.\chi\left(\frac{d u^{e}}{d t}\right)\right|_{t=\zeta_{j}^{e}}}\left(f_{\alpha_{j} \beta_{j-1}}-f_{\alpha_{j} \beta_{j}}\right)+\left[F\left(\alpha_{n}, \beta_{n-1}\right)-F\left(\alpha_{n}, u(t)\right)\right] \quad \dot{u}(t) \leq 0
$$

According to Eq. (17) and Eq. (18), inverse dynamic Preisach model can be written as

$$
\begin{aligned}
u_{1}(t)= & F_{\alpha}^{-1}\left\{\frac { 1 } { 2 } \left[f(t)-\sum_{\mathrm{k}=1}^{n}\left(F\left(\alpha_{k}, \beta_{k-1}\right)-F\left(\alpha_{k}, \beta_{k}\right)\right)-\right.\right. \\
& \left.\left.\sum_{k=1}^{n(t)} \sum_{j=1}^{n_{j}^{k}} \frac{\left.\chi\left(\frac{d u}{d t}\right)\right|_{t=\zeta_{j}}}{\left.\chi\left(\frac{d u^{e}}{d t}\right)\right|_{t=\zeta_{j}^{e}}}\left(f_{\alpha_{j} \beta_{j-1}}-f_{\alpha_{j} \beta_{j}}\right)\right], \beta_{n-1}\right\} \quad \dot{u}(t) \geq 0 \\
u_{1}(t)= & F_{\beta}^{-1}\left\{\alpha_{n}, \frac{1}{2}\left[\sum_{\mathrm{k}=1}^{n-1}\left(F\left(\alpha_{k}, \beta_{k-1}\right)-F\left(\alpha_{k}, \beta_{k}\right)\right)+\right.\right. \\
& \left.\left.\sum_{k=1}^{n(t)-1} \sum_{j=1}^{n_{j}^{k}} \frac{\left.\chi\left(\frac{d u}{d t}\right)\right|_{t=\zeta_{j}}}{\left.\chi\left(\frac{d u^{e}}{d t}\right)\right|_{t=\zeta_{j}^{e}}}\left(f_{\alpha_{j} \beta_{j-1}}-f_{\alpha_{j} \beta_{j}}\right)+2 F\left(\alpha_{n}, \beta_{n-1}\right)-f(t)\right]\right\} \quad \dot{u}(t) \leq 0
\end{aligned}
$$

The input change-rate function $\chi\left(\frac{d u}{d t}\right)$ can be decided by the experimental results.

The Fig. 4 shows the experimental results: with the increasing of input frequency, the maximum value of the output displacement became smaller. According to the experimental results, $\chi\left(\frac{d u}{d t}\right)$ can be expressed by eq. (21) to describe different static and dynamic hysteresis loops.

$$
\chi\left(\frac{d u}{d t}\right)=c_{1}\left(\frac{d u}{d t}\right)^{n}-c_{2}
$$

where $c_{1}, c_{2}$ and $n$ are constant coefficients.

Figs. 3, 4 and 5 show that, due to the complex nonlinearities of the piezoelectric direct drive electro-hydraulic servo valve, there is a big deviation between real output and normalized output. When the users don't need very high positioning precision, the open-loop control can meet the position requirements. This open-loop control mode don't need sensor and the cost is low. Feedforward control based on dynamic Preisach model can effectively overcome the hysteresis effect and improve the control accuracy, but as the result of the unmodeling information, modeling error and the external disturbance, its positioning precision is not very high. Therefore, when the users need high precision, the feedforward control can not meet the requirements, and then the closed-loop feedback control is needed.

\subsection{High Precision Fuzzy Control with Dynamic Preisach Model Feedforward Loop}

To improve the dynamic and steady state performance of the new type of piezoelectric electro-hydraulic valve system, a high precision fuzzy control which is used as feedback loop control is proposed.

Fig. 8 is the structure of the high precision fuzzy control with dynamic Preisach model feedforward loop. This system consists of a dynamic Preisach model feedforward and a high precision fuzzy controller with Lagrange interpolation algorithm. $u_{1}(t)$ is control voltage from the dynamic Preisach model, and it is used to compensate the nonlinearities of 
the piezoelectric direct drive electro-hydraulic servo valve. $u_{2}(t)$ is the control signal from the high precision fuzzy controller.

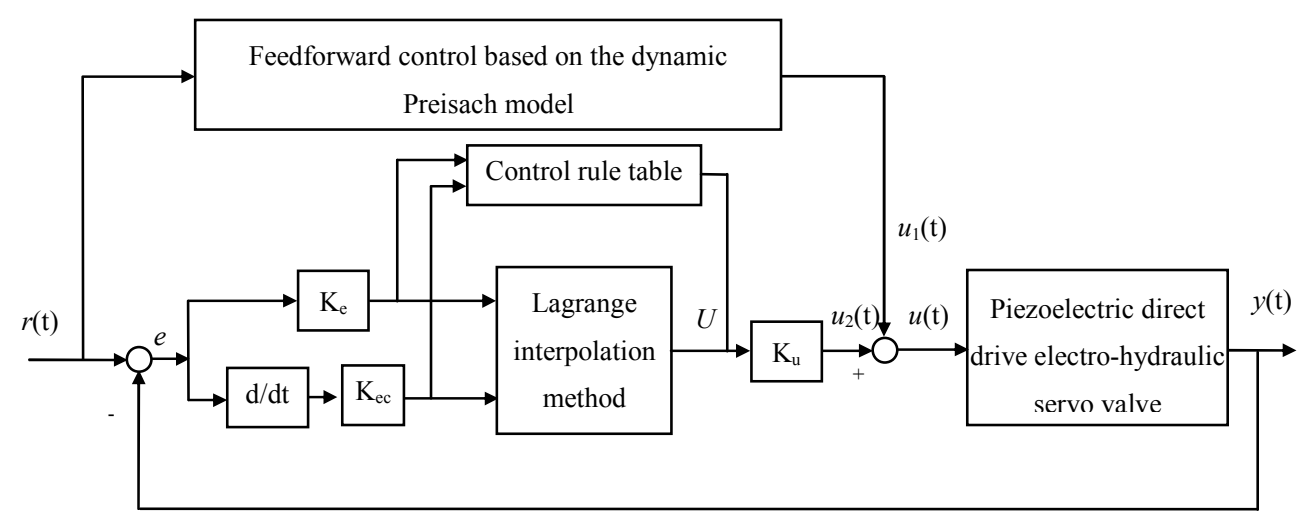

Fig. 8 Structure of the high precision fuzzy control with dynamic Preisach model feedforward loop

The existence of control dead range is the main reason why the controlling precision of fuzzy control based on look-up table algorithm is poor. The key to improving the controlling precision of fuzzy control algorithm based on look-up-table is to eliminate its control dead range ${ }^{(19)}$. Hence, the basic idea of the high precision fuzzy control is: when error or change of error is not equal to 0 , the on-line algorithm of high precision fuzzy control is also based on the look-up table from the off-line design, but when error and change of error are 0 , interpolation algorithm based on look-up table is adopted instead of quantization.

Table 1 Look-up table of Fuzzy control

\begin{tabular}{|c|c|c|c|c|c|c|c|c|c|c|c|c|c|}
\hline \multirow[b]{2}{*}{ error } & \multicolumn{13}{|c|}{ Change of error } \\
\hline & -6 & -5 & -4 & -3 & -2 & -1 & 0 & 1 & 2 & 3 & 4 & 5 & 6 \\
\hline-6 & 7 & 6 & 7 & 6 & 7 & 7 & 7 & 4 & 4 & 2 & 0 & 0 & 0 \\
\hline-5 & 6 & 6 & 6 & 6 & 6 & 6 & 6 & 4 & 4 & 2 & 0 & 0 & 0 \\
\hline-4 & 7 & 6 & 7 & 6 & 7 & 7 & 7 & 4 & 4 & 2 & 0 & 0 & 0 \\
\hline-3 & 7 & 6 & 6 & 6 & 6 & 6 & 6 & 3 & 2 & 0 & -1 & -1 & -1 \\
\hline-2 & 4 & 4 & 4 & 5 & 4 & 4 & 2 & 1 & 0 & 0 & -1 & -1 & -1 \\
\hline-1 & 4 & 4 & 4 & 5 & 4 & 15 & $\overline{1}$ & 0 & 0 & 0 & -1 & -2 & -2 \\
\hline 0 & 4 & 4 & 4 & 5 & 1 & 1 & 0 & -11 & -1 & -1 & -4 & -4 & -4 \\
\hline 1 & 2 & 2 & 2 & 2 & 0 & 1_0_ & $=1$ & $-2 !$ & -4 & -3 & -4 & -4 & -4 \\
\hline 2 & 1 & 2 & 1 & 2 & 0 & -3 & -4 & -4 & -4 & -3 & -4 & -4 & -4 \\
\hline 3 & 0 & 0 & 0 & 0 & -3 & -3 & -6 & -6 & -6 & -6 & -6 & -6 & -6 \\
\hline 4 & 0 & 0 & 0 & -2 & -4 & -4 & -7 & -7 & -7 & -6 & -7 & -6 & -7 \\
\hline 5 & 0 & 0 & 0 & -2 & -4 & -4 & -6 & -6 & -6 & -6 & -6 & -6 & -6 \\
\hline 6 & 0 & 0 & 0 & -2 & -4 & -4 & -7 & -7 & -7 & -6 & -7 & -6 & -7 \\
\hline
\end{tabular}

The adopted interpolation method is Lagrange interpolation method. The Lagrange 
interpolation formula of the bivariate function is:

$$
U=f(e, e c) \approx \sum_{k=0}^{n} \sum_{r=0}^{m} l_{k}(e) \bar{l}_{r}(e c) f\left(e_{k}, e c_{r}\right)
$$

where $e_{\mathrm{k}}$ and $e c_{\mathrm{r}}$ are error and change of error, $k=\{-6,-5, \cdots, 0, \cdots, 6\}$, $r=\{-6,-5, \cdots, 0, \cdots, 6\}$.

$$
\begin{aligned}
& l_{k}(e)=\prod_{t=0, t \neq k}^{n} \frac{e-e_{t}}{e_{k}-e_{t}} \\
& \bar{l}_{r}(e c)=\prod_{t=0, t \neq k}^{m} \frac{e c-e c_{t}}{e c_{r}-e c_{t}}
\end{aligned}
$$

In order to easily realized in the engineering, the piecewise quadratic interpolation is adopted. Supposing that

$$
\begin{aligned}
& e_{t}=e_{o}+i h, \quad i=0,1, \cdots, n \\
& e c_{t}=e c_{o}+j \tau, \quad j=0,1, \cdots, m \\
& h>0, \tau>0
\end{aligned}
$$

When $(e, e c)$ is given, and $(e, e c)$ satisfies:

$$
\begin{aligned}
& e_{t}-\frac{h}{2}<e \leq e_{t}+\frac{h}{2}, \quad 1 \leq i \leq n-1 \\
& e c_{t}-\frac{\tau}{2}<e c \leq e c_{t}+\frac{\tau}{2}, \quad 1 \leq j \leq m-1
\end{aligned}
$$

Then, $\left(e_{k}, e c_{r}\right)(k=i-1, i, i+1, r=j-1, j, j+1)$ should be selected as the interpolation point, the corresponding interpolation polynomial is:

$$
U=f(e, e c) \approx \sum_{k=i-1}^{i+1} \sum_{r=j-1}^{j+1} l_{k}(e) \bar{l}_{r}(e c) f\left(e_{k}, e c_{r}\right)
$$

where

$$
\begin{aligned}
& l_{k}(e)=\prod_{t=i-1, t \neq k}^{i+1} \frac{e-e_{t}}{e_{k}-e_{t}}, \quad k=i-1, \quad i, \quad i+1 \\
& \bar{l}_{r}(e c)=\prod_{t=i-1, t \neq r}^{j+1} \frac{e c-e c_{t}}{e c_{r}-e c_{t}}, \quad r=j-1, \quad j, \quad j+1
\end{aligned}
$$

If $e \leq e_{1}-\frac{h}{2}$ or $e>e_{n-1}+\frac{h}{2}$, then in eq. (27): $i=1$ or $i=n-1$, if $e c \leq e c_{1}-\frac{\tau}{2}$ or $e c>e c_{m-1}+\frac{\tau}{2}$, then in eq. (27): $j=1$ or $j=m-1$.

Finally, $u_{2}(t)=k_{u} U$ is output control of high precision fuzzy controller. $u_{1}(t)$ and $u_{2}(t)$ are added up to get the final control voltage $u(t)$ which controls the piezoelectric direct drive electro-hydraulic servo valve.

\section{Experimental Study}

The output precision of the piezoelectric direct drive electro-hydraulic servo valve system is significantly reduced by nonlinear effects such as hysteresis and creep nonlinearities. To demonstrate the effectiveness of the proposed control method, a series of experiments are performed on the piezoelectric direct drive electro-hydraulic servo valve 
system.

The working parameters in the experiment are as follows: the range of position sensor's feedback signal: $0 \sim 10 \mathrm{~V}$, oil pressure is $7 \mathrm{Mpa}$, flowrate is $0 \sim 4 \mathrm{~L} / \mathrm{min}$.

Figs. 9 and 10 are the step response curves when adopting fuzzy control and the proposed control with the step-function signal from 1 to $7 \mathrm{~V}$. Both control algorithms' step responses have not overshoot. When adopting common fuzzy control, the system can reach the steady state in $5.48 \mathrm{~ms}$; when adopting the proposed control method, the system can reach the steady state in $4.77 \mathrm{~ms}$. It can be seen from the figures that the high-precise fuzzy control method with the dynamic Preisach model in feedforward loop has a better control effect than common fuzzy control.

ANO SOKKI CF-5200 MULTI-PURPOSE FFT ANALYZER

4.0KHz A: DC/31.6ur B: DC/3.16 Ur

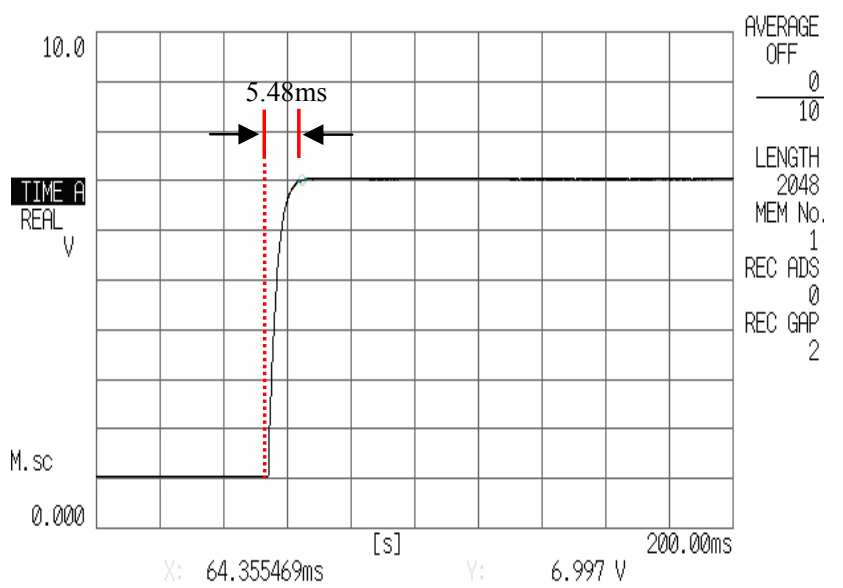

Fig. 9 Step response curve under the fuzzy control method

\section{ANO SOKKI CF-5200 MULTI-PURPOSE FFT ANALYZER}

4.0KHz $\quad A: D C / 10.0 \mathrm{Vr} \quad B: D C / 3.16 \mathrm{Vr}$

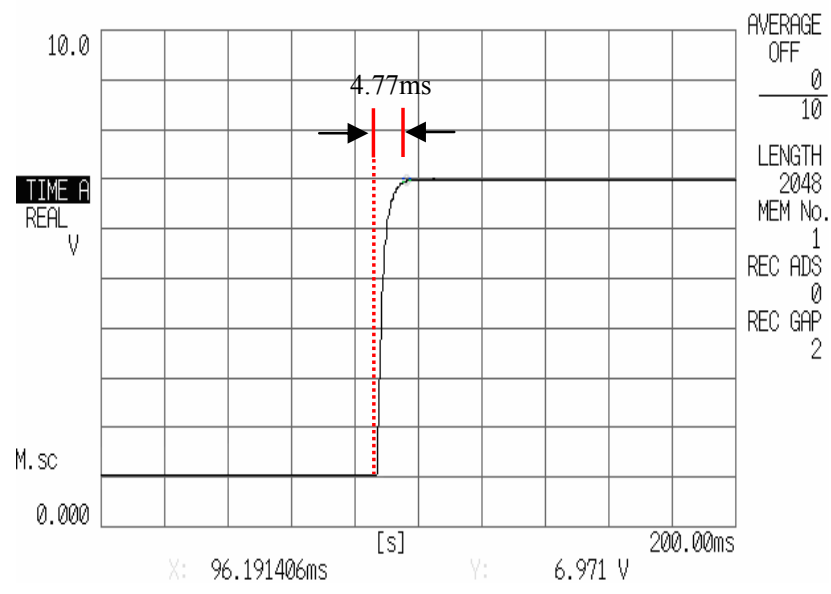

Fig. 10 Step response curve under the proposed control method

Figs. 11 show the frequency response characteristic curves of system. In the figure, the blue broken line curve is amplitude frequency characteristic under the conventional fuzzy control method; the blue real line is amplitude frequency characteristic under proposed control method. It can be seen from figure that the amplitude frequency bandwidth is 410 
$\mathrm{Hz}$ under the conventional fuzzy control method; the amplitude frequency bandwidth is 470 $\mathrm{Hz}$ under proposed control method. It can show that the proposed control method can provide better dynamic characteristic than the conventional fuzzy control method.

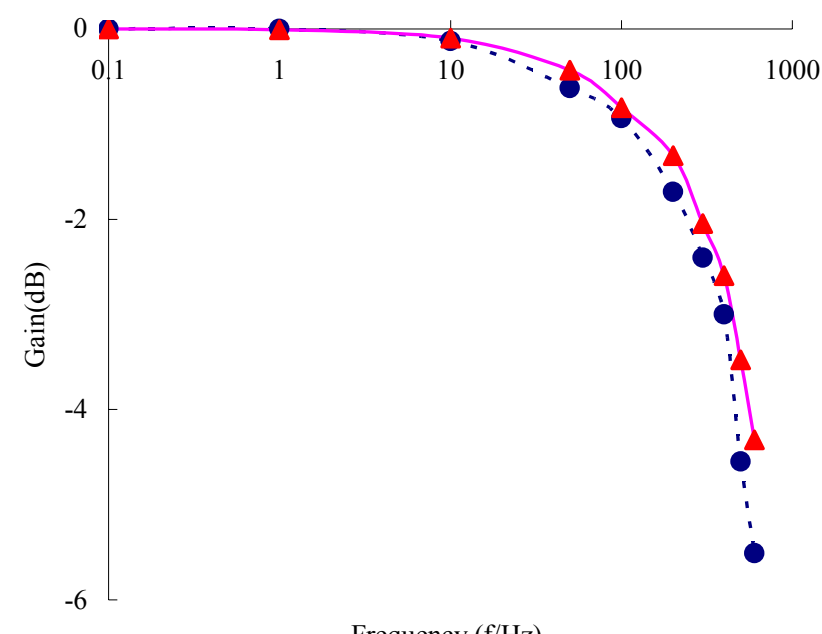

Frequency (f/Hz)

Fig. 11 Frequency response characteristic curves under the conventional fuzzy control method and the proposed control method

Fig. 12 shows the hysteresis loop output curve of the system under the proposed control method. It is calculated that the maximum output hysteresis is less than $0.43 \mu \mathrm{m}$ and the hysteresis loop is $0.86 \%$. The experimental results show that the phenomenon of the hysteresis loop and creep nonlinearity can be eliminated well.

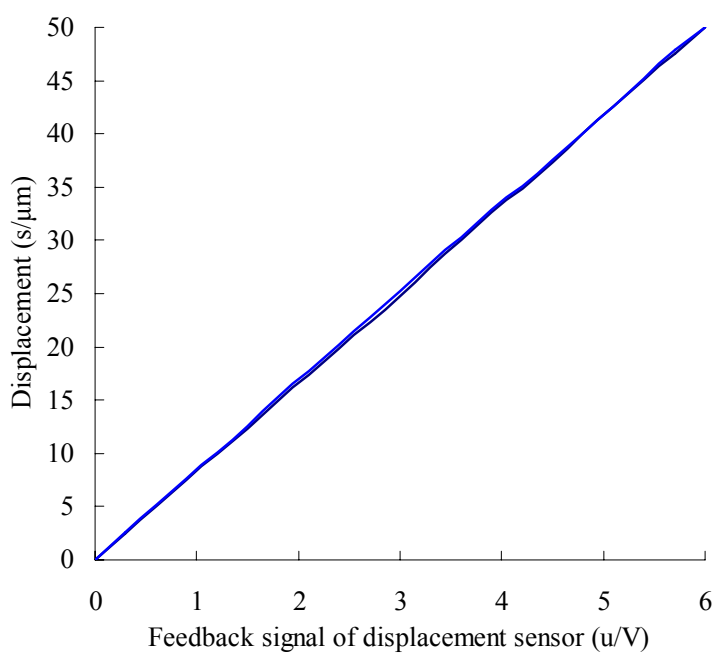

Fig. 12 Output curve under the proposed control method

\section{Conclusions}

By use of high frequency characteristic of piezoelectric actuator, a piezoelectric direct drive electro-hydraulic servo valve is proposed. Its structure and work principle are introduced. In the experimental research, the hysteresis and creep nonlinearities existing in the designed piezoelectric direct drive electro-hydraulic servo valve system are found. These nonlinearities can seriously influence the output precision and dynamic characteristic 
of system. In order to solve the hysteresis and the creep nonlinear problems of the piezoelectric direct drive electro-hydraulic servo valve system, a high-precise fuzzy control method with the dynamic Preisach model in feedforward loop is proposed in this paper. This high precision fuzzy controller adopts Lagrange interpolation method of bivariate function, and it can obtain continuous variable control quantity, eliminate the quantization error completely.

The experimental results show that the high precision fuzzy control method can implement the control accurately and has a more sensitive response than the conventional fuzzy control method. The maximum output hysteresis is less than $0.43 \mu \mathrm{m}$ and the hysteresis loop is $0.86 \%$ under the proposed control method. Contrasted to conventional fuzzy control method, the amplitude frequency bandwidth can be improved $14.63 \%$ with the proposed control method. The experimental results show that the proposed control method can resolve the phenomenon of the hysteresis loop and creep, and improve the frequency bandwidth of system.

Acknowledgments: This research is partially supported by National Natural Science Foundation of China (Grant No.: 51105170), Program of Science and Technology Development Plan of Jilin province of China (Grant No.: 201105015).

References

(1) Nobuhiko, H., An Open-Loop Positioning Method for Reduction of Influence of Nonlinearities in Piezoelectric Actuator,Journal of Advanced Mechanical Design, Systems, and Manufacturing, Vol.5,No.1(2011), pp.70-79.

(2) Trabia, M.B., Yim, W. and Saadeh, M., Modeling of Hysteresis and Backlash Within a Smart Fin With a Piezoelectric Actuator,Journal of Intelligent Material Systems and Structures, Vol.22,No.11(2011), pp.1161-1176.

(3) Boettcher, U., Callafon, R.A.D. and Talke F.E., Modeling and Control of a Dual Stage Actuator Hard Disk Drive,Journal of Advanced Mechanical Design, Systems and Manufacturing, Vol.4,No.1(2010), pp.107-118.

(4) Shinichi, Y., An Ultra Fast-Acting Electro-Hydraulic Digital Valve and High-Speed Electro-Hydraulic Servo Valve Using Multilayered PNT Elements,Proceeding of the second JHPS International Symposium on Fluid Power, (1993), pp.121-130.

(5) Lindler, J.E. and Anderson, E.H., Piezoelectric Direct Drive Servovalve,Proceeding of the SPIE International Conference on Industrial and Commercial Applications of Smart Structures Technologies, No.4698-5(2002-3), pp.488-496.

(6) Chun, G.S. and Han, K.B., Micromachined Valves Based on Multilayer Piezoelectric Actuators,Electronics Letters, Vol.44,No.18(2008), pp.1058-1059.

(7) Bang, Y.B., Joo, C.S., Lee, K.I., Hur, J.W. and Lim, W.K., Development of a Two-Stage High Speed Electrohydraulic Servovalve Systems Using Stack-Type Piezoelectric Elements,Proceedings of the 2003 IEEE/ASME international Conference on Advanced intelligent Mechatronics, No.1(2003-7), pp.131-136.

(8) Karunanidhi, S. and Singaperumal, M., Mathematical Modelling and Experimental Characterization of a High Dynamic Servo Valve Integrated with Piezolelctric Actuator,Proceedings of the Institution of Mechanical Engineers, Part I: Journal of Systems and Control Engineering, 
No.4(2010-6), pp.419-435.

(9) Ma, Y.H., Mao, J.Q. and Zhang, Z., On Generalized Dynamic Preisach Operator with Application to Hysteresis Nonlinear Systems,IEEE Transactions on Control Systems Technology, Vol.19,No.6(2011), pp.1527-1533.

(10) Rakotondrabe, M., Bouc-Wen Modeling and Inverse Multiplicative Structure to Compensate Hysteresis Nonlinearity in Piezoelectric Actuators,IEEE Transactions on Automation Science and Engineering, Vol.8,No.2(2011), pp.428-431.

(11) Ishikiriyama, Y. and Morita, T., Improvement of Self-sensing Piezoelectric Actuator Control Using Permittivity Change Detection,Journal of Advanced Mechanical Design, Systems, and Manufacturing, Vol.4,No.1(2010), pp.143-149.

(12) Ge, P. and Jouaneh, M., Tracking Control of a Piezoceramic Actuator,IEEE Transactions on Control Systems and Technology, Vol.4,No.3(1996), pp.209-216.

(13) Zhou, M.L., Tian, Y.T., Gao, W. and Yang, Z.G., High precise control method for a new type of piezoelectric electro-hydraulic servo valve,Journal of Central South University of Technology, Vol.14,No.6(2007), pp.832-837.

(14) Wong, P.K., Xu, Q.S., Vong, C.M. and Wong, H.C., Rate-Dependent Hysteresis Modeling and Control of a Piezostage Using Online Support Vector Machine and Relevance Vector Machine,IEEE Transactions on Industrial Electronics, Vol.59,No.4(2012), pp.1988-2001.

(15) Song, G., Zhao, J.Q., Zhou, X.Q. and Abreu-Garcia J.A.D., Tracking Control of a Piezoceramic Actuator with Hysteresis Compensation Using Inverse Preisach Model,IEEE/ASME Transactions on Mechatronics, Vol.10,No.2(2005), pp.198-209.

(16) Hwang, C.L., Chen, Y.M. and Jan, C., Trajectory Tracking of Large-Displacement Piezoelectric Actuators Using a Nonlinear Observer-Based Variable Structure Control,IEEE Transactions on Control Systems Technology, Vol.13,No.1(2005), pp.56-66.

(17) Ge, P. and Jouaneh, M., Modeling Hysteresis in Piezoceramic Actuators,Precision Engineering, Vol.17,No.3(1995), pp.211-221.

(18) Yu, Y., Xiao, Z., Naganathan, N.G. and Dukkipati, R.V., Dynamic Preisach Modelling of Hysteresis for The Piezoceramic Actuator System,Mechanism and Machine Theory, Vol.37,No.1(2002), pp.75-89.

(19) Zhang Z.K., Chang J., A fuzzy control algorithm with high controlling precision, Fuzzy Sets and Systems, Vol.140,No.2(2003), pp.375-385. 